\title{
Sparse Reconstruction of Multi-Window Time-Frequency Representation Based on Hermite functions
}

\author{
Branka Jokanović, Moeness G. Amin, and Yimin D. Zhang \\ Center for Advanced Communications, Villanova University, Villanova, PA 19085, USA
}

\begin{abstract}
Multi-window spectrograms offer higher energy concentration in contrast to the traditional single-window spectrograms. However, these quadratic time-frequency distributions were not introduced to deal with randomly undersampled signals. This paper applies sparse reconstruction techniques to provide time-frequency representations of nonstationary signals using the Hermite functions as multiple windows, under randomly sampled or missing data. The multi-window sparse reconstruction approach improves energy concentration by utilizing the common local sparse frequency support property across the different employed windows.
\end{abstract}

Keywords: Time-frequency representation, Hermite function, multi-window spectrogram, multi-measurement vector, random undersampling

\section{INTRODUCTION}

Time-frequency distributions (TFDs) are a powerful tool for the analysis of signals with time-varying spectrum [1], [2]. These signals are frequently encountered in various areas, such as radar, seismology and biomedicine [3]. Numerous investigations have been made in order to find the optimal time-frequency representation (TFR). However, even though different TFDs have been proposed, none has provided an ideal TFR for all signal categories.

The simplest linear TFR is the short-time Fourier transform (STFT). This transform is obtained by projecting the signal over the Fourier basis defined by a sliding window in time. The square modulus of STFT is spectrogram. The main issue of spectrogram is that its resolution in the time-frequency domain is dependent on the window size and shape. This drawback limits the use of a single-window spectrogram, motivating the introduction of different approaches employing a set of windows [4]- [5]. The discrete prolate spheroidal sequence (DPSS) and the Hermitian functions are well-known approaches for the generation of such windows. The DPSS is an optimal sequence for providing maximum spectral concentration [6]. The Hermite functions are also attractive due to their simplicity, recursion, and performance comparison with DPSS [6], [7].

Traditional bilinear or quadratic time-frequency analysis is not suited to deal with incomplete data. Over the past few years, compressive sensing (CS), an approach that addresses the issue of missing samples, has become an area of growing interest [8]- [10]. Even though CS has been extensively studied in many areas, few researchers have addressed the sparsity in the time-frequency domain [11]- [16].

In this paper, we consider multi-window sparse reconstruction of TFR of signals with missing samples. In order to do so, we realize that spectrograms obtained from a set of Hermite functions share similar support in the time-frequency domain. This inspires, from a sparse reconstruction perspective, the application of multimeasurement vector model [17] to provide desirable multi-window TFR from compressed data observations. Unlike spectrograms, these reconstructions techniques are not Fourier based and as such resolution does not explicitly depend on the employed data window length or neither it is bounded by Rayleigh limit. Attempts to enhance resolution in the time-frequency domain have so far applied the parametric approach in lieu of Fourier transform [18], [19]. In contrast to the approach in [20], there is no need for designing a reduced interference distribution kernel from which windows are computed. In our case, the window functions and their properties are known in advance.

The paper is organized as follows. In Section 2, we review the multi-window spectrogram concept and the definition of the Hermite functions. The recovery of multi-window TFR using the multiple-measurement vector approach is presented in Section 3, whereas simulation results are presented in Section 4. Conclusion is given in Section 5.

Further author information: E-mail: branka.jokanovic@villanova.edu 


\section{MULTI-WINDOW TIME-FREQUENCY SIGNAL REPRESENTATION}

A multi-window spectrogram, $S P E C_{M W}(t, \omega)$, can be defined as the weighted sum of $L$ spectrograms $S P E C_{l}(t, \omega)$, $l=0,1 \ldots, L-1$, expressed as $[21]$,

$$
\operatorname{SPEC}_{M W}(t, \omega)=\sum_{l=0}^{L-1} c_{l}(t) S P E C_{l}(t, \omega),
$$

where the weights are determined by the coefficients $c_{l}(t)$. Each spectrogram in (1) is obtained by applying a Hermite function of $l$-th order $\Phi_{l}(\tau)$ to the windowed signal $s(t)$ and computing the Fourier transform, i.e.,

$$
\operatorname{SPEC}_{l}(t, \omega)=\left|\int_{\tau} s(t+\tau) \Phi_{l}(\tau) e^{-\jmath \omega \tau} d \tau\right|^{2} .
$$

The Hermite functions of the 0th and 1st order are respectively defined as,

$$
\begin{aligned}
& \Phi_{0}(t)=\frac{1}{\sqrt[4]{\pi}} e^{-t^{2} / 2}, \\
& \Phi_{1}(t)=\frac{\sqrt{2} t}{\sqrt[4]{\pi}} e^{-t^{2} / 2} .
\end{aligned}
$$

By using recursion, Hermite functions of the higher order can be efficiently computed as

$$
\Phi_{l}(t)=t \sqrt{\frac{2}{l}} \Phi_{l-1}(t)-\sqrt{\frac{l-1}{l}} \Phi_{l-2}(t), \forall l \geq 2 .
$$

Figure 1 shows the Hermite functions from the 0th to the 5th order. By using this set of functions as windows in spectrogram, we can significantly improve signal power concentration in the time-frequency domain, which is demonstrated in Figure 2 for a signal consisting of two sinusoidal frequency modulated (FM) components.

A higher number of Hermite functions will provide better concentration in the time-frequency domain. For a signal $A(t) e^{j \Psi(t)}$, the optimal number $L$ depends on the signal phase order. Additionally, if $A(t)$ is constant over a window, i.e., $A(t)=A(t+\tau)$, the weighting coefficients $c_{l}(t)$ can assume constant value [21]. In this paper, we use six Hermite functions and set constant values of the weighting coefficients.

\section{RECONSTRUCTION OF THE MULTI-WINDOW TFR}

In this section, we address the issue of estimating the multi-window TFR of a signal vector $\mathbf{x}=[x(1), \ldots, x(N)]^{T}$ which has missing samples in the time domain, where $(\cdot)^{T}$ denotes transpose. This signal can be represented as the sum of the original signal $s(n)$ and the set of missing samples at random positions $n_{i}$, i.e.,

$$
x(n)=s(n)-\sum_{n_{i}} \delta\left(n-n_{i}\right) s(n) .
$$

The multi-window spectrogram of $\mathbf{x}$ using $L$ Hermite functions is defined as,

$$
\operatorname{SPEC}_{M W}(n, k)=\sum_{l=0}^{L-1} c_{l}\left|\sum_{m}(s(n+m)-v(n+m)) \Phi_{l}(m) e^{-\jmath 2 \pi k m / N}\right|^{2},
$$

where

$$
v(n)=\sum_{n_{i}} \delta\left(n-n_{i}\right) s(n) .
$$

Due to the missing samples in the time domain, the resulting TFR suffers from noise-like artifacts. These artifacts clutter the desired TFR and obscure the signal time-frequency signatures. In order to reduce the artifacts and 

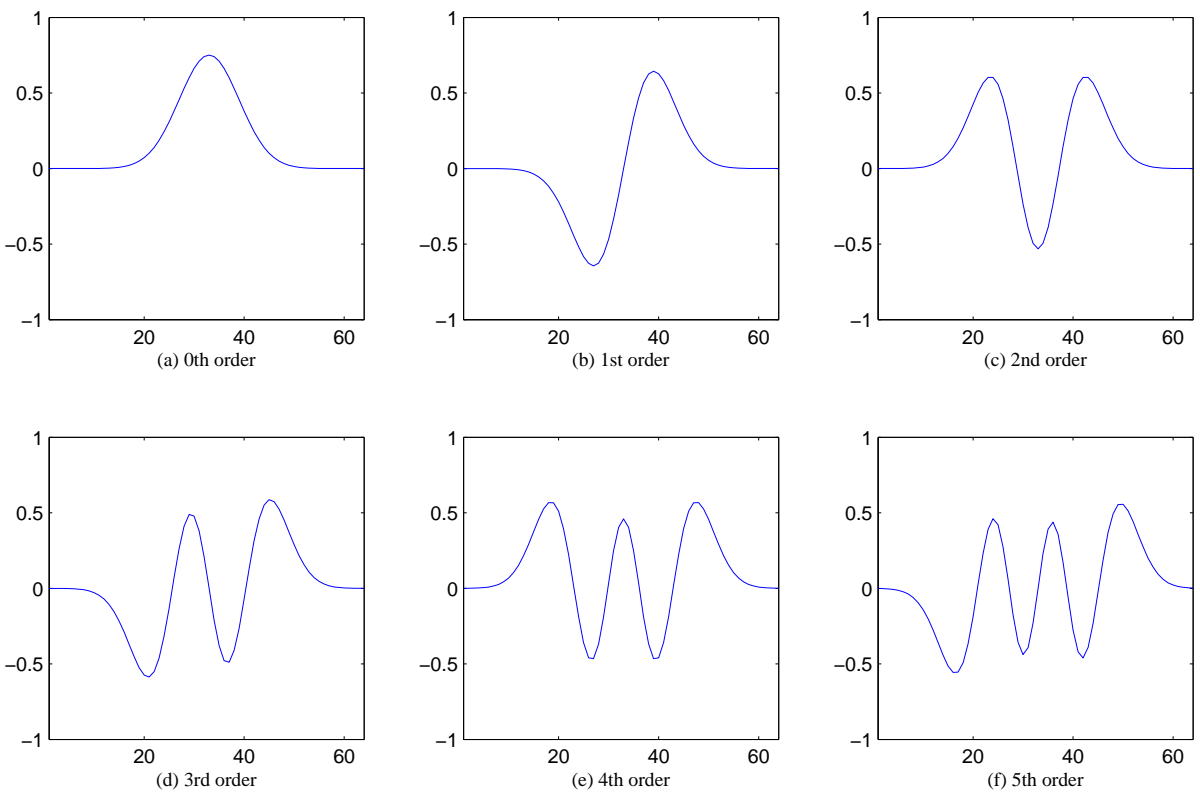

Figure 1. Hermite functions from the 0th to the 5th order.

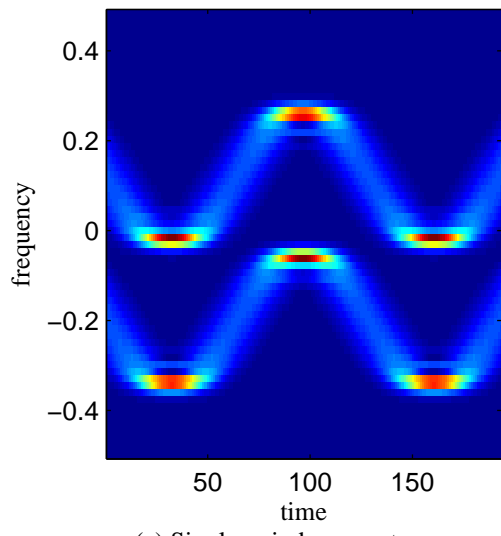

(a) Single-window spectrogram

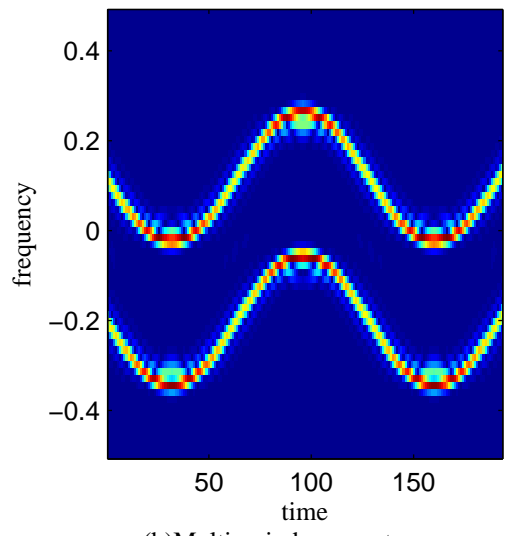

(b)Multi-window spectrogram

Figure 2. TFRs of a multi-component signal consisting of two sinusoidal FM components. 

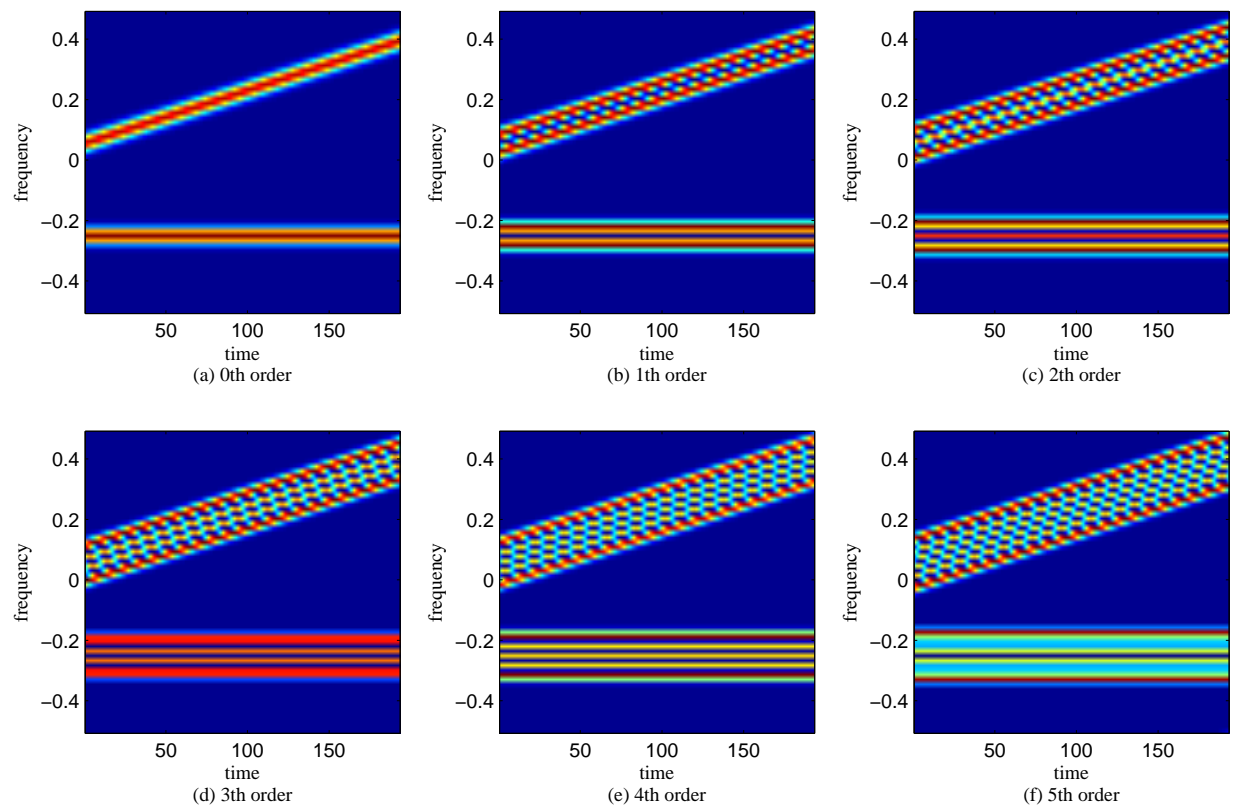

Figure 3. Spectrograms of a multi-component signal consisting of a chirp and a sinusoid. Hermite functions from 0th to 5 th order are employed as windows.

obtain proper signal TFR, we utilize sparse recovery approach. Namely, for the majority of nonstationary signals, the TFRs can be considered sparse, since the energy is localized over a small bandwidth at a given time instant. This property invites the applications of sparse recovery methods for time-frequency signal representations. More specifically, in the case of multi-window spectrogram, we observe that all spectrograms in (7) share similar frequency support. This property is evident in Figure 3, where Hermite function based spectrograms of a multicomponent signal consisting of a chirp and a sinusoid are shown. This feature translates into common sparsity support within the compressive sensing paradigm. We incorporate the common sparsity support in the underlying problem through the multiple vector measurements approach where multiple windows are used [17]. For the $l$ th Hermite function window we have the linear model,

$$
\mathbf{y}_{n}^{l}=\mathbf{W}_{n} \mathbf{f}_{n}^{l},
$$

where $\mathbf{y}_{n}^{l}$ is the measurement vector, $\mathbf{f}_{n}^{l}$ is the sparse vector, while $\mathbf{W}_{n}$ is a partial Fourier matrix at time $n$ and is the same for $l=1, \ldots, L$. After obtaining $\mathbf{f}_{n}^{l}, \forall l$, under the common sparsity property, we can estimate multiwindow TFR. If we denote $\mathbf{D}$ as the estimated multi-window TFR, then each column of $\mathbf{D}$, which corresponds to time $n$, is obtained as,

$$
\mathbf{d}_{n}=\sum_{l=0}^{L-1} c_{l}\left|\mathbf{f}_{n}^{l}\right|^{2} .
$$

It should be noted that (10) can also be used when each $\mathbf{f}_{n}^{l}$ is reconstructed separately, i.e., without imposing the joint sparsity support. However, $\mathbf{f}_{n}^{l}$ obtained in this manner do not, in general, share the sparsity support. Hence, the result is different and less attractive than the case when all vectors are provided simultaneously. It is noticed that the use of multi-window approach in TFR provides two advantages in contrast to the single-window approach. The multi-window approach overcomes the resolution limitations of spectrogram. Additionally, multiple windows produce multiple measurement sets which improve the sparse vector estimation. 


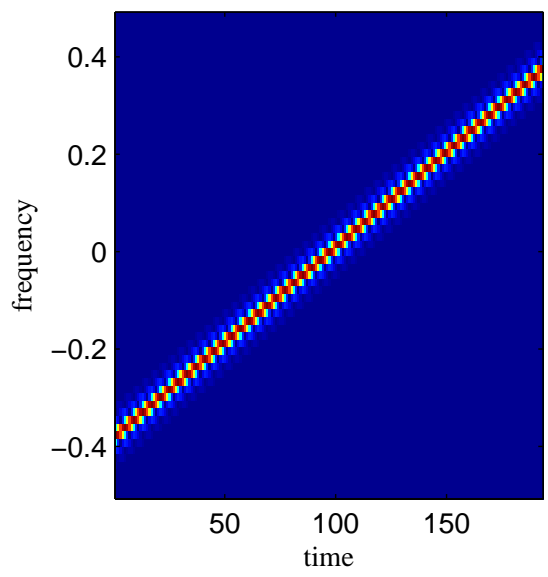

(a) Full data

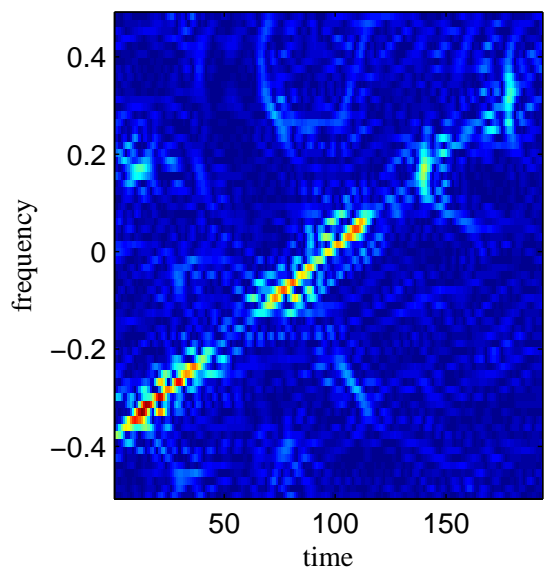

(b) Incomplete data

Figure 4. Multi-window spectrogram of a linear FM signal.

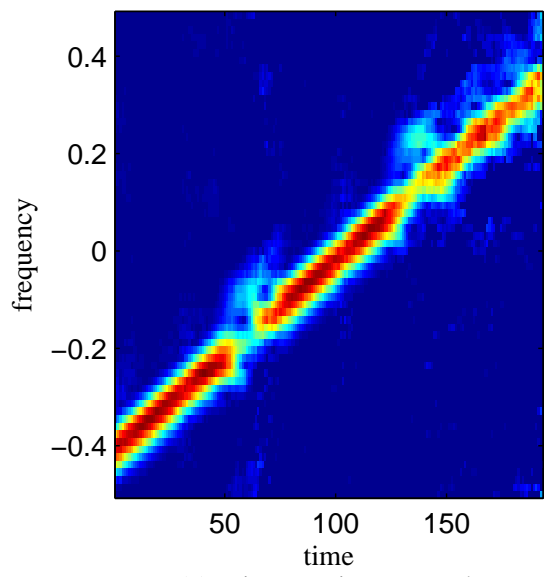

(a) Joint sparsity approach

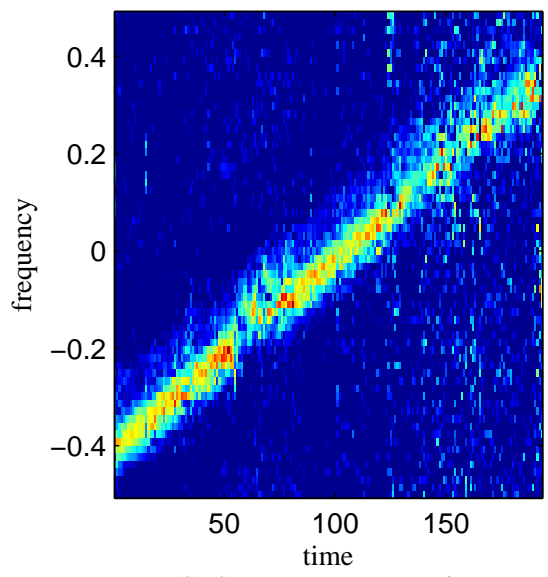

(b) Separate reconstruction

Figure 5. Multi-window TFRs using the joint sparsity approach and separate reconstruction.

\section{SIMULATION RESULTS}

In this section, we evaluate the multi-window approach using a set of Hermite functions for the sparse recovery of the TFR. The results demonstrate that multiple windows are a powerful tool for enhancing the energy localization and in conjunction with the joint sparsity support constraint, they can be used for enhanced time-frequency distributions.

\subsection{Mono-component signal}

We first consider a linear FM signal, i.e., chirp (Figure 4(a)). The signal is randomly undersampled, with only $30 \%$ out of 256 samples present. The high number of missing samples degrades the multi-window spectrogram (Figure 4(b)). The result obtained using the M-FOCUSS algorithm, i.e., assuming joint sparsity support is shown in Figure 5(a). As a comparison, we also include result obtained by reconstructing each $\mathbf{f}_{n}^{l}$ in (10) separately using orthogonal matching pursuit (OMP), i.e, without imposing the joint sparsity support (Figure 5(b)). The superiority of the former approach is evident. 


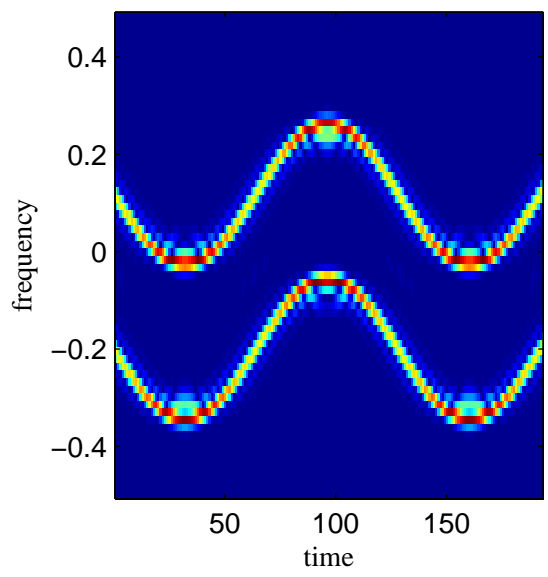

(a) Full data

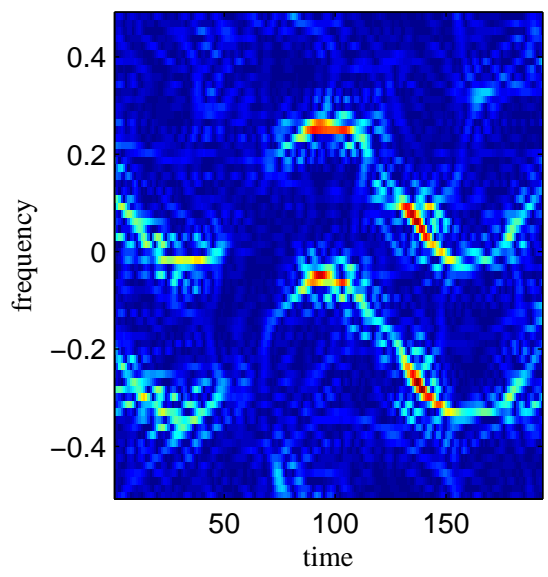

(b) Incomplete data

Figure 6. Multi-window spectrogram of two sinusoidal FM signals.

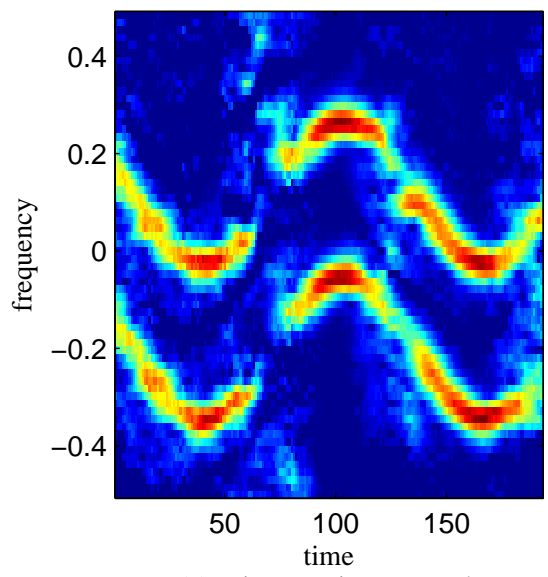

(a) Joint sparsity approach

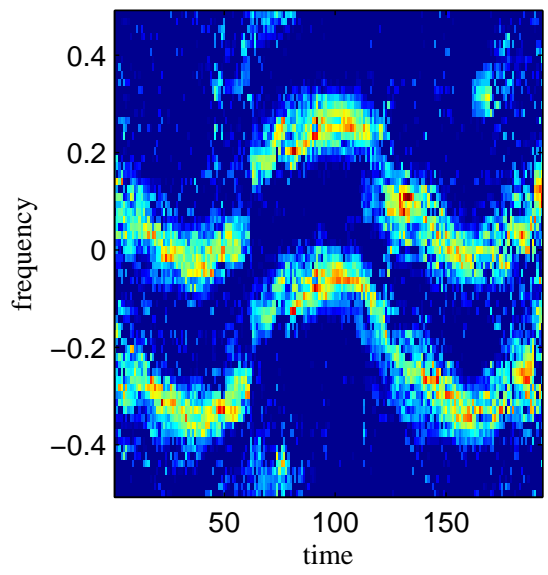

(b) Separate reconstruction

Figure 7. Multi-window TFRs using the joint sparsity approach and separate reconstruction.

\subsection{Multi-component signal}

In the next example, we consider multi-component signal. The signal being observed consists of a two sinusoidal FM signals. $50 \%$ of 256 data samples are randomly missing. As in the previous case, we notice the distortion in the multi-window spectrogram caused by missing samples in the time domain (Figure 6). Significant improvement is obtained by using the joint sparsity approach, as shown in Figure 7(a). Further, the TFR is efficiently estimated and contains low artifacts when compared to the case of reconstructing each TFR separately, as shown in Figure $7(\mathrm{~b})$.

\section{CONCLUSION}

In this paper, we provided time-frequency signal representations in the case of incomplete data using Hermite functions and compressive sensing. Multi-window spectrogram is a well-known technique for overcoming the limited resolution of the traditional spectrogram; however, it was not designed to deal with missing samples. By using the fact that spectrograms corresponding to different Hermite functions share similar sparse support, we showed that the use of multiple windows within the sparse reconstruction framework is beneficial for two reasons; it provides high energy localization, i.e., resolution, and improves the sparse signature recovery. 


\section{REFERENCES}

[1] B. Boashash, Time Frequency Analysis. Gulf Professional Publishing, 2003.

[2] L. Cohen, Time-Frequency Analysis: Theory and Applications. Prentice-Hall, Inc., 1995.

[3] P. Flandrin, M. Amin, S. McLaughlin, and B. Torresani, "Time-frequency analysis and applications [from the guest editors]," IEEE Signal Process. Magazine, vol. 30, no. 6, pp. 19-150, 2013.

[4] G. Fraser and B. Boashash, "Multiple window spectrogram and time-frequency distributions," in Proc. IEEE ICASSP, vol. 4, 1994, pp. IV-293.

[5] M. Hansson, "Multiple window decomposition of time-frequency kernels using a penalty function for suppressed sidelobes," in Proc. IEEE ICASSP, 2006, pp. 2883-2886.

[6] Y. Xu, S. Haykin, and R. J. Racine, "Multiple window time-frequency distribution and coherence of EEG using slepian sequences and hermite functions," IEEE Trans. Bio. Engin., vol. 46, no. 7, pp. 861-866, 1999.

[7] I. Orović, N. Žarić, S. Stanković, and M. Amin, "A multiwindow time-frequency approach based on the concepts of robust estimate theory," pp. 3584-3587, 2011.

[8] E. J. Candès and M. B. Wakin, "An introduction to compressive sampling," IEEE Signal Process. Magazine, vol. 25, no. 2, pp. 21-30, 2008.

[9] D. L. Donoho, "Compressed sensing," IEEE Trans. Inf. Theory., vol. 52, no. 4, pp. 1289-1306, 2006.

[10] J. A. Tropp and A. C. Gilbert, "Signal recovery from random measurements via orthogonal matching pursuit," IEEE Trans. Inf. Theory, vol. 53, no. 12, pp. 4655-4666, 2007.

[11] P. Flandrin and P. Borgnat, "Time-frequency energy distributions meet compressed sensing," IEEE Trans. Signal Process., vol. 58, no. 6, pp. 2974-2982, 2010.

[12] M. G. Amin (ed.), Compressive Sensing for Urban Radar. CRC Press, 2014.

[13] L. Stankovic, I. Orovic, S. Stankovic, and M. Amin, "Compressive sensing based separation of nonstationary and stationary signals overlapping in time-frequency," IEEE Trans. Signal Process., vol. 61, no. 18, pp. 4562-4572, Sept 2013.

[14] B. Jokanović, M. Amin, and S. Stanković, "Instantaneous frequency and time-frequency signature estimation using compressive sensing," in Proc. SPIE, vol. 871418, 2013.

[15] Y. D. Zhang, M. G. Amin, and B. Himed, "Reduced interference time-frequency representations and sparse reconstruction of undersampled data," in Proc. European Signal Proc. Conf., 2013.

[16] L. Stankovic, S. Stankovic, and M. Amin, "Missing samples analysis in signals for applications to Lestimation and compressive sensing," Signal Processing, vol. 94, pp. 401-408, 2014.

[17] S. F. Cotter, B. D. Rao, K. Engan, and K. Kreutz-Delgado, "Sparse solutions to linear inverse problems with multiple measurement vectors," IEEE Trans. Signal Process., vol. 53, no. 7, pp. 2477-2488, 2005.

[18] M. G. Amin and W. J. Williams, "High spectral resolution time-frequency distribution kernels," IEEE Trans. Signal Process., vol. 46, no. 10, pp. 2796-2804, 1998.

[19] B. Barkat and B. Boashash, "A high-resolution quadratic time-frequency distribution for multicomponent signals analysis," IEEE Trans. Signal Process., vol. 49, no. 10, pp. 2232-2239, 2001.

[20] M. G. Amin, Y. D. Zhang, and B. Jokanović, "Time-frequency signature reconstruction from random observations using multiple measurement vectors," in Proc. IEEE ICASSP, 2014.

[21] F. Çakrak and P. J. Loughlin, "Multiwindow time-varying spectrum with instantaneous bandwidth and frequency constraints," IEEE Trans. Signal Process., vol. 49, no. 8, pp. 1656-1666, 2001. 